\title{
Article
}

\section{Phthalates Induce Androgenic Effects at Exposure Levels That Can Be Environmentally Relevant in Humans}

Tian, Meiping, Liu, Liangpo, Wang, Heng, Wang, Xiaofei, Martin, Francis L, Zhang, Jie, Huang, Qingyu and Shen, Heqing

Available at https://clok.uclan.ac.uk/22302/

Tian, Meiping, Liu, Liangpo, Wang, Heng, Wang, Xiaofei, Martin, Francis L orcid iconORCID: 0000-0001-8562-4944, Zhang, Jie, Huang, Qingyu and Shen, Heqing (2018) Phthalates Induce Androgenic Effects at Exposure Levels That Can Be Environmentally Relevant in Humans. Environmental Science \& Technology Letters, 5 (5). pp. 232-236.

It is advisable to refer to the publisher's version if you intend to cite from the work. http://dx.doi.org/10.1021/acs.estlett.8b00138

For more information about UCLan's research in this area go to http://www.uclan.ac.uk/researchgroups/ and search for <name of research Group>.

For information about Research generally at UCLan please go to http://www.uclan.ac.uk/research/

All outputs in CLoK are protected by Intellectual Property Rights law, including Copyright law. Copyright, IPR and Moral Rights for the works on this site are retained by the individual authors and/or other copyright owners. Terms and conditions for use of this material are defined in the policies page. 


\section{Supporting Information}

\section{Phthalates Induce Androgenic Effects at Exposure Levels that can be Environmentally Relevant in Humans}

Meiping Tian ${ }^{\mathrm{a}, \mathrm{b}}$, Liangpo Liu ${ }^{\mathrm{a}}$, Heng Wang ${ }^{\mathrm{a}}$, Xiaofei Wang ${ }^{\mathrm{a}}$, Francis L. Martinc $^{\mathrm{c}}$, Jie Zhang ${ }^{\mathrm{a}}$, Qingyu Huang ${ }^{\mathrm{a}}$, Heqing Shen ${ }^{\mathrm{a}^{*}}$

${ }^{a}$ Key Lab of Urban Environment and Health, Institute of Urban Environment, Chinese Academy of Sciences, Xiamen 361021, China;

${ }^{b}$ College of Resources and Environment, University of Chinese Academy of Sciences, Beijing 100049, China; and,

${ }^{c}$ School of Pharmacy and Biomedical Sciences, University of Central Lancashire (UCLan), Preston PR1 2HE, UK

*Corresponding Author: Heqing Shen, Institute of Urban Environment, Chinese Academy of Sciences, 1799 Jimei Road, Xiamen 361021, China; Telephone/Fax: (86)592-6190771; E-mail: hqshen@iue.ac.cn 


\section{SI-1: Supplementary to male population study}

Chemicals and Stock Solutions. $\mathrm{C}_{13}$ and $\mathrm{C}_{12}$ mono-ester phthalate monomethyl phthalate (MMP), monoethyl phthalate (MEP), monobutyl phthalate (MBP), mono-2ethylhexyl phthalate (MEHP), monobenzyl phthalate (MBzP), mono-2-ethyl-5hydroxyhexyl phthalate (MEHHP), mono-2-ethyl-5-carboxypentyl (MECPP) and mono-2-ethyl-5-oxohexyl phthalate (MEOHP) were purchased from CIL (Cambridge Isotope Laboratory Ins, USA). The steroid hormone standards of androstenedione (ASD) and testosterone were purchased from Dr. Ehrenstorfer GmbH (Germany), the isotope $\mathrm{D}_{3}$-testosterone was purchased from Cerilliant (Promochem, Wesel, Germany). Escherichia coli $\beta$-glucuronididase was purchased from Sigma-Aldrich, USA. Oasis HLB solid phase extraction (SPE) cartridges (60 mg, $3 \mathrm{~mL}$, Waters, USA) were purchased from Waters Corporation (Milford, MA). All other chemicals were analytical grade.

Deconjugating of the glucuronidated phthalate metabolite. Aliquots of $1 \mathrm{~mL}$ urine were buffered to $\mathrm{pH}=6.5$ by sodium acetate $(1 \mathrm{~mL}, 0.1 \mathrm{M} \mathrm{NaAc}$ adjusted with $1 \mathrm{M}$ $\mathrm{HCl}$ ), and spiked with a mixture of isotope labeled phthalate monoesters as the internal standards $(20 \mu \mathrm{L}, 50 \mathrm{ng} / \mathrm{mL})$. $\beta$-Glucuronidase $(10 \mu \mathrm{L}, 200$ units $/ \mathrm{mL})$ was added as deconjugation enzyme and 4-methylumbelliferyl glucuronide $(20 \mu \mathrm{L}, 80 \mathrm{ng} / \mathrm{mL})$ was added as the control indicator of the enzyme activity. The samples were incubated at $37^{\circ} \mathrm{C}$ for $90 \mathrm{~min}$ to deconjugate the glucuronidated phthalate metabolites and the reaction was stop through the addition of $1 \mathrm{~mL}$ of $3.6 \mathrm{M}$ phosphoric acid. 
SPE Purification. The column was preconditioned with acetonitrile $(3 \mathrm{~mL})$ and phosphate buffer $\left(3 \mathrm{~mL}, \mathrm{pH}=2,0.14 \mathrm{M} \mathrm{NaH}_{2} \mathrm{PO}_{4}\right.$ in $\left.0.85 \% \mathrm{H}_{3} \mathrm{PO}_{4}\right)$ in sequence. The treated urine was loaded onto the cartridge at a rate of $1 \mathrm{~mL} / \mathrm{min}$. Then it was rinsed with $0.1 \mathrm{M}$ formic acid $(2 \mathrm{~mL})$ and $\mathrm{H}_{2} \mathrm{O}(1 \mathrm{~mL})$ and dried with a gentle airflow. The analytes were eluted with acetonitrile $(3 \mathrm{~mL})$ followed by ethyl acetate $(2 \mathrm{~mL})$ at 1 $\mathrm{mL} / \mathrm{min}$. The eluate was evaporated to dryness under a gentle stream of nitrogen at $45^{\circ} \mathrm{C}$ and the dried residue was re-suspended in $200 \mu \mathrm{L}$ of $60 \%$ acetonitrile $(0.1 \%$ acetic acid $)$ until further analysis.

HPLC-ESI-MS/MS analysis. The target compounds were separated by the LC-20A prominence (Shimadzu, Kyoto, Japan) high-performance liquid chromatography (HPLC) equipped with the Phenomenex ${ }^{\circledR}$ (Torrance, CA, USA) guard column (Phenyl, $4.0 \mathrm{~mm} \times 2.0 \mathrm{~mm}$ internal diameter) and separation column (Luna Phenyl-hexyl, 100 $\mathrm{mm} \times 2.0 \mathrm{~mm}$ internal diameter, $3.0 \mu \mathrm{m}$ particle size). Mobile phase A was $0.1 \%$ acetic acid in water and $\mathrm{B}$ was $0.1 \%$ acetic acid in acetonitrile. Table S1 shows the HPLC gradient program. The mobile phase flow rate was set at $0.25 \mathrm{~mL} / \mathrm{min}$, and the column temperature was set at $30^{\circ} \mathrm{C}$. The total run time for each injection was $20 \mathrm{~min}$ and the injection volume was $20 \mu \mathrm{L}$.

The target compounds were ionized by electrospray ionization (ESI) and detected by the tandem mass spectrometry (MS/MS) (Applied Biosystems, Concord, Ontario, Canada). The mass specific detection was achieved using a MDS Sciex 3200 Q TRAP triple quadrupole linear ion trap mass spectrometer equipped with a TurboIonSpray ESI source and Analyst Software version 1.5 (Applied Biosystems, Concord, Ontario, 
Canada). ESI-MS/MS was performed at unit mass resolution in multiple reaction monitoring (MRM) under the negative ion mode with the following parameters: source temperature (TEM) $550^{\circ} \mathrm{C}$, ion spray voltage (IS) $-4500 \mathrm{~V}$, curtain gas (CUR) $10 \mathrm{psi}$ (46 kPa of nitrogen), ion source gas 1 (GS1) $40 \mathrm{psi}$ (276 kPa of nitrogen), ion source gas 2 (GS2) $40 \mathrm{psi}$ (276 kPa of nitrogen), collision gas pressure (nitrogen) medium [corresponds to value of 3 (arbitrary scale of 1-12)], and dwell time of $50 \mathrm{~ms}$ per channel. Optimization of the compound-dependent MS/MS parameters was performed with phthalate metabolites standards via direct injection into the mass spectrometer using a syringe pump at a flow rate of $10 \mu \mathrm{L} / \mathrm{min}$. Optimal settings for compounddependent MS/MS parameters are shown in Table S2.

The Absolute Recovery of SPE Extraction. The phthalate metabolite's standard curve regression equation and absolute recoveries during SPE extraction were calculated (Table S3 and S4). Considering the background analytes in the urine, we used the labeled internal standard to track the SPE recovery instead of the native compounds. One of the paired spiking samples was added the labeled internal standards before SPE

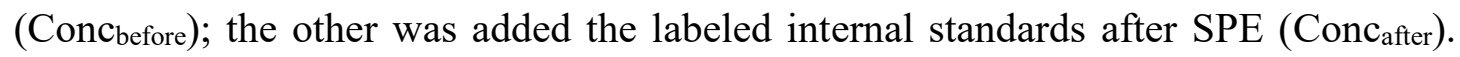
The SPE recovery was calculated as: SPE recovery $=\left(\right.$ Conc $_{\text {after }} /$ Conc $\left._{\text {before }}\right) \times 100 \%$. Because of the recoveries in the urine expression step are close to hundred percent for the analytes, the absolute recoveries of the whole sample preparation procedure should be comparable with the reported SPE extraction recoveries for adult urine analysis ${ }^{1}$. In case of the low SPE recovery, the stable isotope-labeled compounds as internal standards had been used to correct the possible matrix effects and to improve method 
accuracy and precision.

Table S1. Mobile Phase Gradient Program ${ }^{\mathrm{a}}$

\begin{tabular}{lccccccccc}
\hline Time (min) & 0 & 0.3 & 1.3 & 8.0 & 12 & 12.5 & 13.5 & 14.0 & 20.0 \\
\hline $\mathbf{A}(\%)$ & 5 & 5 & 40 & 20 & 20 & 0 & 0 & 5 & 5 \\
$\mathbf{B}(\%)$ & 95 & 95 & 60 & 80 & 80 & 100 & 100 & 95 & 5
\end{tabular}

${ }^{\mathrm{a}} \mathrm{A}: 0.1 \%$ acetic acid in water and $\mathrm{B}: 0.1 \%$ acetic acid in acetonitrile. 
Table S2. Transitions and MS/MS Conditions for Each Analyte

\begin{tabular}{|c|c|c|c|c|c|c|c|c|}
\hline Analytes & Parent & Daughter & Dwell & DP & $\mathbf{E P}$ & CEP & CE & CX \\
\hline MMP & 179.0 & 107.0 & 50.0 & - & - & -10.0 & - & 0.0 \\
\hline MMP. ${ }^{13} \mathrm{C}_{4}$ & 183.0 & 110.0 & 50.0 & -33.0 & -7.0 & -17.7 & -14.0 & 0.0 \\
\hline MEP & 193.0 & 121.0 & 50.0 & -30.0 & -7.0 & -10.0 & -16.0 & 0.0 \\
\hline $\operatorname{MEP}{ }^{13} \mathrm{C}_{4}$ & 197.0 & 79.0 & 50.0 & -33.0 & -7.0 & -18.3 & -33.0 & 0.0 \\
\hline MBP & 221.0 & 77.0 & 50.0 & -30.0 & -7.0 & -12.0 & -20.0 & 0.0 \\
\hline $\mathrm{MBP}{ }^{13} \mathrm{C}_{4}$ & 225.0 & 79.0 & 50.0 & -35.0 & -7.0 & -19.5 & -28.0 & 0.0 \\
\hline MBzP & 255.0 & 107.0 & 50.0 & -30.0 & -7.0 & -15.0 & -19.0 & 0.0 \\
\hline $\operatorname{MBzP}-{ }^{13} \mathrm{C}_{4}$ & 259.0 & 107.0 & 50.0 & -35.0 & -7.0 & -20.0 & -21.0 & 0.0 \\
\hline МЕНР & 277.0 & 134.0 & 50.0 & -30.0 & -7.0 & -15.0 & -22.0 & 0.0 \\
\hline MEHP. ${ }^{13} \mathrm{C}_{4}$ & 281.0 & 137.0 & 50.0 & -45.0 & -7.0 & -21.8 & -22.0 & 0.0 \\
\hline МЕОНР & 291.0 & 121.0 & 50.0 & -40.0 & -4.0 & -15.0 & -35.0 & 0.0 \\
\hline МЕОНР- & 295.0 & 124.0 & 50.0 & -36.0 & -7.0 & -22.4 & -27.0 & 0.0 \\
\hline МЕСРP & 307.0 & 159.0 & 50.0 & -40.0 & -6.0 & -16.0 & -20.0 & 0.0 \\
\hline МЕСРP- & 311.0 & 159.0 & 50.0 & -40.0 & -5.0 & -20.0 & -18.0 & 0.0 \\
\hline МЕEHP & 293.0 & 145.0 & 50.0 & -35.0 & -7.5 & -22.0 & -28.0 & 0.0 \\
\hline МЕНHР- & 297.0 & 124.0 & 50.0 & -40.0 & -6.5 & -16.0 & -28.0 & 0.0 \\
\hline
\end{tabular}

DP: declustering potential [V]; EP: entrance potential [V]; CEP: cell entrance potential [V]; CE: collision energy [V]; CXP: cell exit potential [V]. 
Table S3. The Phthalate Metabolite's Absolute Recoveries in SPE Extraction Step.

\begin{tabular}{lcccc}
\hline \multirow{2}{*}{ Metabolites } & \multicolumn{3}{c}{$50 \mathrm{ng} / \mathrm{ml} / \mathrm{ml}$} & \\
\cline { 2 - 5 } & $\begin{array}{c}\text { Mean Recovery } \\
(\%)\end{array}$ & RSD (\%) & $\begin{array}{c}\text { Mean Recovery } \\
(\%)\end{array}$ & RSD (\%) \\
\hline MMP & 141 & 6.91 & 95.1 & 9.56 \\
MEP & 120 & 149 & 95.7 & 6.90 \\
MBP & 88.9 & 3.7 & 97.8 & 14.6 \\
MBzP & 89.0 & 10.5 & 89.4 & 10.5 \\
MEHP & 117 & 13.5 & 95.3 & 4.53 \\
MEOHP & 112 & 12.3 & 108 & 0.8 \\
MECPP & 90.9 & 7.0 & 91.5 & 4.9 \\
MEEHP & 104.4 & 5.7 & 103.6 & 4.4 \\
\hline
\end{tabular}

Table S4. Standard Curve Regression Equation.

\begin{tabular}{ccccc}
\hline Metabolites & Range $(\mu \mathrm{g} / \mathrm{L})$ & Regression & $\mathrm{R}^{2}$ & LOD $(\mu \mathrm{g} / \mathrm{L})$ \\
\hline MMP & $0.2 \sim 200$ & $\mathrm{Y}=0.0237 \mathrm{X}+0.0424$ & 0.996 & 0.21 \\
MEP & $0.2 \sim 200$ & $\mathrm{Y}=0.0179 \mathrm{X}+0.0153$ & 0.999 & 0.45 \\
MBP & $0.2 \sim 200$ & $\mathrm{Y}=0.0122 \mathrm{X}+0.0176$ & 0.994 & 0.50 \\
MBzP & $0.2 \sim 200$ & $\mathrm{Y}=0.0735+0.0044$ & 0.994 & 0.31 \\
MEHP & $0.2 \sim 200$ & $\mathrm{Y}=0.0172 \mathrm{X}+0.011$ & 0.999 & 0.37 \\
MEOHP & $0.2 \sim 200$ & $\mathrm{Y}=0.0128 \mathrm{X}+0.0002$ & 0.992 & 0.37 \\
MECPP & $0.2 \sim 200$ & $\mathrm{Y}=0.0087 \mathrm{X}+0.0025$ & 0.997 & 0.37 \\
MEEHP & $0.2 \sim 200$ & $\mathrm{Y}=0.0174 \mathrm{X}+0.0028$ & 0.998 & 0.37 \\
\hline
\end{tabular}



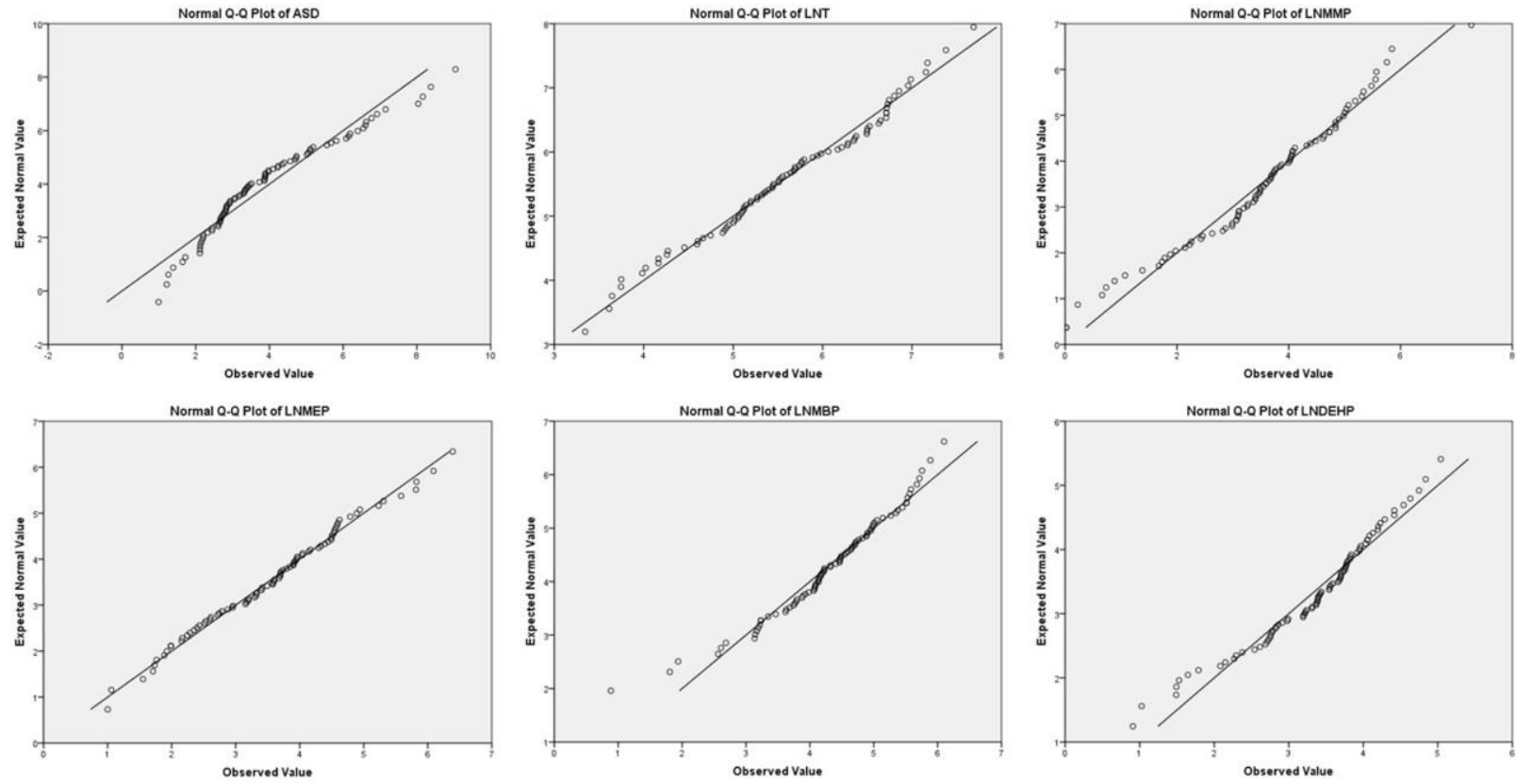

Figure S1. Normal Q-Q plots of the data steroid hormones and phthalate metabolites.

LNT, LNMMP, LNMEP, LNMBP and LNDEHP are the log-transformed testosterone, MMP, MEP, MBP and $\mathrm{DEHP}$, respectively. 

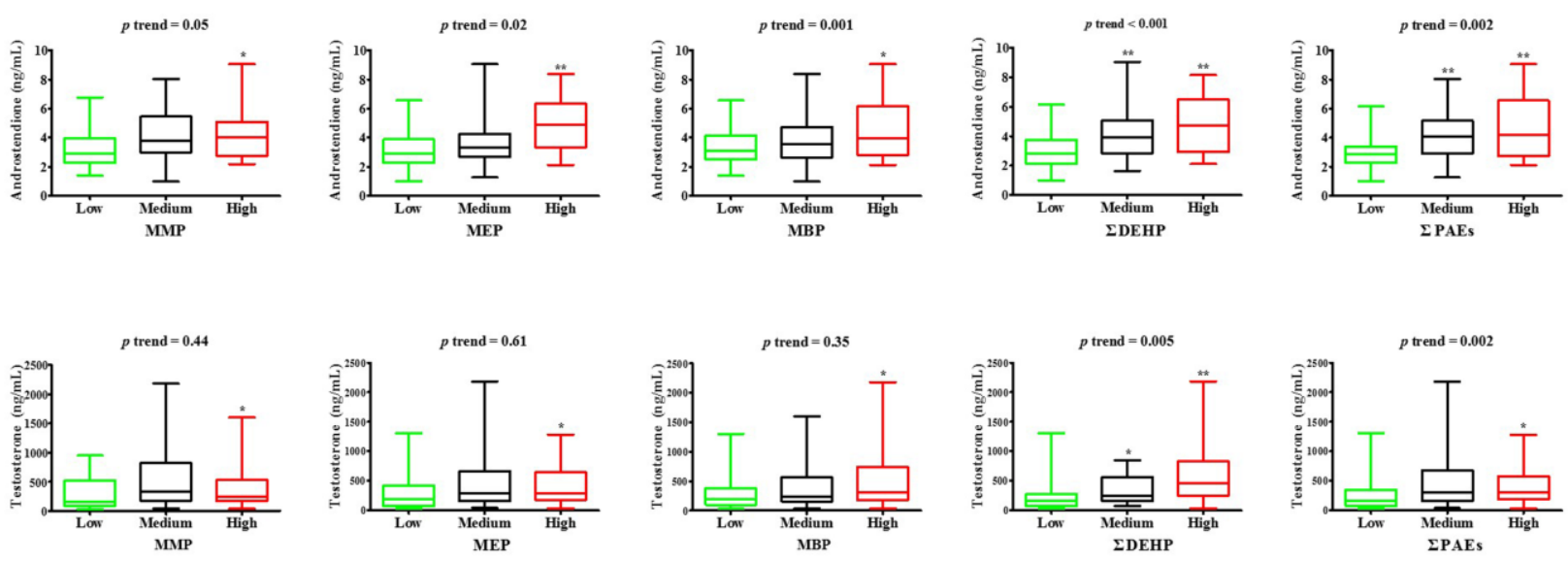

Figure S2. Levels of urine steroid hormones grouped by tertiles of PAEs.

Low $=1^{\text {st }}$ tertile PAEs, medium $=2^{\text {nd }}$ tertile PAEs, and high $=3^{\text {rd }}$ tertile PAEs; the box and-whisker plot shows the median, $75^{\text {th }}$ and $25^{\text {th }}$ percentiles, and maximum and minimum values, respectively. All models are adjusted for BMI, age, plastic use, smoking and alcohol intake. Also shown are $p$-trends of steroid hormone across the tertile of PAEs. DDEHP was the mass sum of DEHP individual metabolites (MEHP, MEOHP, MECPP and MEHHP). ¿PAEs was the mass sum of PAEs individual metabolite (MMP, MEP, MBP, MEHP, MEOHP, MECPP and MEHHP). 
Table S5. Multivariable-Adjusted Linear Regression of the PAEs' Prediction to Steroid Androgen Variations

\begin{tabular}{lllllll}
\hline & & MMP & MEP & MBP & $\sum$ DEHP & $\sum$ PAEs \\
\hline \multirow{2}{*}{ Androstendione } & Coefficient & $\mathbf{0 . 3 3}$ & $\mathbf{0 . 2 8}$ & $\mathbf{0 . 3 5}$ & $\mathbf{0 . 5 5}$ & $\mathbf{0 . 4 4}$ \\
\cline { 2 - 2 } & $95 \% \mathrm{CI}$ & $\mathbf{0 . 0 7 , 0 . 5 8}$ & $\mathbf{0 . 0 2 , \mathbf { 0 . 5 5 }}$ & $\mathbf{0 . 1 1 , \mathbf { 0 . 6 0 }}$ & $\mathbf{0 . 3 1 , \mathbf { 0 . 7 9 }}$ & $\mathbf{0 . 1 9 , 0 . 7 0}$ \\
& $P$-value & $\mathbf{0 . 0 1}$ & $\mathbf{0 . 0 4}$ & $\mathbf{0 . 0 0 6}$ & $<\mathbf{0 . 0 0 1}$ & $\mathbf{0 . 0 0 1}$ \\
& Coefficient & 0.13 & 0.17 & 0.22 & $\mathbf{0 . 4 6}$ & 0.21 \\
\multirow{2}{*}{ Testosterone } & $95 \% \mathrm{CI}$ & $-0.15,0.42$ & $-0.13,0.47$ & $-0.06,0.50$ & $\mathbf{0 . 1 8 , 0 . 7 4}$ & $-0.08,0.51$ \\
& $P$-value & 0.36 & 0.26 & 0.12 & $\mathbf{0 . 0 0 2}$ & 0.15 \\
\hline
\end{tabular}

All models are adjusted for age, BMI and lifestyle habit, including smoking, alcohol intake and plastic usage. Variable ln-transformed in statistical analysis. 


\section{SI-2: In vitro study showed environment relevant PAEs induced biphasic steroidogenesis effect in MLTC-1}

\section{Introduction}

The concept of integrating human observations with laboratory tests comes from the facts both human study and laboratory test can address the metabolic phenotypes in blood and urine or in biotas of in vitro or in vivo. The hypothesis is environmental stimulation induced any effect will finally translate to metabolic layer change. Therefore, to march the metabolite alteration in human with the metabolic effect in toxicological model would improve the communication between the two disciplines (Figure S3) herein.

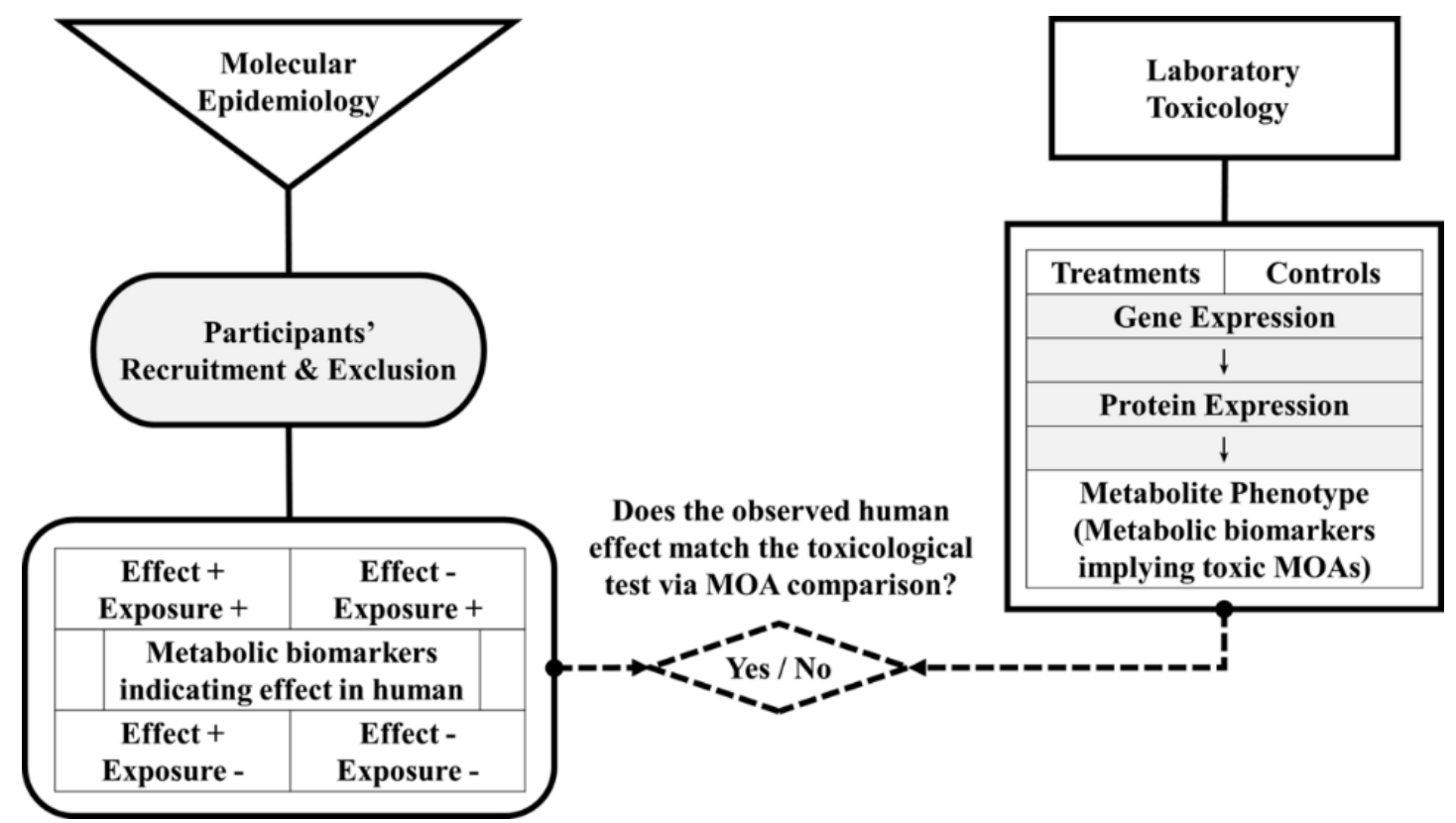

Figure S3. Conceptual workflow towards integrating human observations with laboratory tests by matching the metabolic phenotypes.

$"+"=$ present; "-" = absent; MOA = mode of action. On the concept of coordinated metabolic trends, ASD and testosterone alterations in the MLTC-1 model would support the related observations in human. 
Chemicals and Stock Solutions. The diester phthalate standards dimethyl phthalate (DMP), diethyl phthalate (DEP), dibutyl phthalate (DBP), di(2-ethylhexyl) phthalate (DEHP) and dibenzyl phthalate (DBzP) were purchased from TCI (TCI Europe, Belgium, Brussels); both target diester phthalates and monoester phthalates are shown in Table S6. Dimethyl sulfoxide (DMSO) was purchased from Sigma-Aldrich Chemical Co. (St. Louis, Mo. USA); human chorionic gonadotrophin (hCG) was obtained from PROSPECT (Ness-Ziona, Israel). Standards for monoester phthalate and androgen analysis have listed in SI-1 it together with Escherichia coli ßglucuronididase information. All other chemicals were analytical grade.

Cell culture and MTT assay. The MLTC-1 cell line was obtained from the Cell Bank of the Chinese Academy of Sciences (Shanghai, China) and cultured in RPMI-1640 medium (Gibco BRL, Grand Island, NY) supplemented with 100 unit/mL penicillin, 100 unit/mL streptomycin and 10\% (v/v) foetal bovine serum (Hyclone, USA). The cells were grown at $37^{\circ} \mathrm{C}$ with $5 \% \mathrm{CO}_{2}$ in a humidified incubator (SANYO, Japan). MLTC-1 cells were incubated with serial concentrations of mixtures of or individual phthalates for $48 \mathrm{~h}$. All experiments were performed in triplicate. Selected phthalates (Table S6) were dissolved in DMSO. Cell viability was evaluated by the MTT proliferation assay. In brief, cells were plated at a density of $1.5 \times 10^{4}$ per well in $96-$ well plates. After 48-h incubation in the presence of different selected phthalates at a wide range of doses (i.e., 0-2000 $\mu \mathrm{M}), 50 \mu \mathrm{L}$ MTT $(5 \mathrm{mg} / \mathrm{mL})$ was added to each well and then the cells were incubated for another $4 \mathrm{~h}$ at $37^{\circ} \mathrm{C}$. Cell populations that were not treated with phthalates served as negative controls. After removing culture medium, 
$150 \mu \mathrm{L}$ DMSO was added to each well and oscillation was performed for $10 \mathrm{~min}$. Absorbance was determined at $490 \mathrm{~nm}$ and results presented as percentage of values relative to untreated controls. Only non-cytotoxic concentrations were used for the following experiments.

MLTC-1 cell phthalate exposure. The first set of phthalate mixture exposure experiments were designed to verify the phthalate doses depend mode of actions and support the observed association of male population phthalate exposure with sex hormones. MLTC-1 Leydig cells were exposed to a combination of phthalates from 0.1 to $100 \mu \mathrm{M}$. MLTC-1 cells were exposed to DMSO vehicle control, the four levels of equimolar diester phthalate mixtures (i.e., $0.1,1,10$ or $100 \mu \mathrm{M}$ of DMP, DEP, DBP, DBzP and DEHP in mixtures), the four levels of equimolar monoester phthalate mixtures (i.e., 0.1, 1, 10 or $100 \mu \mathrm{M}$ of MMP, MEP, MBP, MBzP or MEHP in mixtures), and the one level of diester phthalate mixture $(100 \mu \mathrm{M}$ DMP, DEP, DBP, DBzP or DEHP in mixtures, respectively) plus carboxylic ester hydrolase of 15 units (SigmaAldrich, USA) to facilitate PAEs hydrolysis. To distinguish phthalate species associated sexual hormone disruption, MLTC-1 cells were treated with both DMSO vehicle control and two concentrations (i.e., 10 or $100 \mu \mathrm{M}$ ) of individual diester phthalate congeners in the second set of individual exposure experiments. The individual phthalates were DMP, DEP, DBP, DBzP and DEHP, respectively. The third set of experiments were set to verify the dose-dependent effect of DBP. MLTC-1 cells were exposed to either DMSO vehicle control or DBP at 5, 10, 25, 50 or $100 \mu \mathrm{M}$. After 48-h phthalate treatment, the culture medium was collected for phthalate metabolites 
analysis, the cells were washed with PBS and serum-free medium. After these steps, the cells were further stimulated with hCG for $4 \mathrm{~h}$ in serum-free medium with $0.1 \%$ BSA, then this medium was collected for ASD and testosterone determination.

\section{PAE metabolites and steroid hormones measurements in cell culture medium}

Phthalate metabolites and steroid hormones in cell culture medium were detected using LC-ESI-MS/MS following the protocol like in urine sample, which have be described in SI-1. 
Table S6. Detailed Information on ten Selected Phthalates

\begin{tabular}{|c|c|c|c|c|}
\hline CAS & Abbreviation & Purity (\%) & Molecular weight & Structure \\
\hline $131-11-3$ & DMP & 99.5 & 194.2 & \\
\hline $84-66-2$ & DEP & 99.0 & 222.2 & \\
\hline $84-74-2$ & DBP & 98.4 & 278.3 & \\
\hline $523-31-9$ & $\mathrm{DBzP}$ & 99.9 & 318.3 & \\
\hline $117-81-7$ & DEHP & 99.5 & 390.6 & \\
\hline $4376-18-5$ & MMP & 97.0 & 180.2 & \\
\hline $2306-33-4$ & MEP & 98.7 & 194.2 & \\
\hline $131-70-4$ & $\mathrm{MBP}$ & 97.4 & 222.2 & \\
\hline $2528-16-7$ & $\mathrm{MBzP}$ & 99.8 & 295.3 & \\
\hline $4376-20-9$ & MEHP & 99.9 & 278.3 & \\
\hline
\end{tabular}




\section{RESULTS}

\section{PAEs exposure range and Leydig cell viability}

Cytotoxicity results limit the PAEs dosage setting. For monoester phthalates: MMP, MEP and MBP generated no significant effects on cell viability under the experimental conditions employed; MEHP at $1000 \mu \mathrm{M}$ or $2000 \mu \mathrm{M}$ reduced cell viability when compared to control $(p<0.001)$ after 48 -h treatment; and, MBzP decreased cell viability at $2000 \mu \mathrm{M}(p<0.001)$ after 48 -h treatment. For diester phthalates: DMP, DEP or DBzP generated no significant effect on cell viability under the experimental conditions employed; and, DBP or DEHP significantly inhibited cell viability at $1000 \mu \mathrm{M}$ or $2000 \mu \mathrm{M}(p<0.01)$ (Figure S4). To ensure cell viability, subsequent experiments used doses of 0.1 to $100 \mu \mathrm{M}$ for each congener; even for the mixture treatment condition of the $100-\mu \mathrm{M}$ group, good cell survival was ensured.
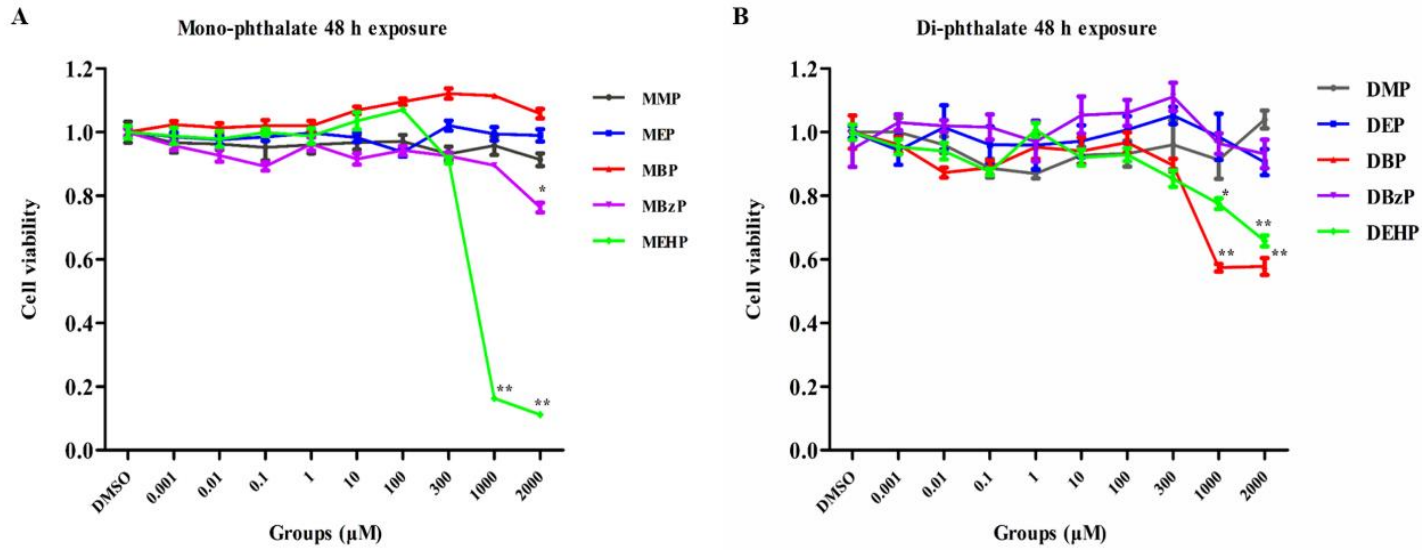

Figure S4. The viability of MLTC-1 cells exposed to various concentrations of mixed monoester phthalate (A) and diester phthalate (B) for $48 \mathrm{~h}$. The values are expressed as the means $( \pm$ SEM) of survival (\% of control cells). Data were analysed by one-way ANOVA with Tukey's multiple comparisons test. Asterisks above columns indicate a significant difference in comparison to the control, $* p \leq 0.05, * * p \leq 0.01$. 


\section{Diester PAEs metabolism in Leydig cells}

Given PAEs' ready human metabolism, understanding their biotransformation in the MLTC-1 model is also important for risk assessment. After 48-h diester PAEs treatment, MMP, MEP, MBP, MEHP and MBzP were quantified. As shown in Figure S5, at low-dose $(0.1-10 \mu \mathrm{M})$ exposure, MLTC-1 cells can readily remove one of the alkyl chains from DMP, DEP or DBP and generate the corresponding monoesters of MMP, MEP or MBP, respectively. DEHP appears harder to hydrolyse into MEHP under these experimental conditions. Generally, the hydrolysis rates decrease when PAE (DMP, DEP, DBP or DEHP) concentrations are increased. To confirm the side chain-specific hydrolysis, exogenous snail-derived esterase was added together with $100 \mu \mathrm{M}$ diester PAEs. The results indicate that the esterase significantly facilitates the hydrolysis of short straight alkyl chain DMP, DEP or DBP $(p<0.001)$, while the effects are not observed for the branched alkyl chain DEHP and aromatic alkyl chain DBzP $(p>0.05)$ (Table S7). MLTC-1 cells hydrolyse PAEs in the following order: DMP > DEP $\geq$ DBP $>$ DEHP, while DBzP cannot be hydrolysed into MBzP in MLTC-1 cells at any concentration. Although many oxidative metabolites of MEHP are observed in human urine ${ }^{1-3}$, major species like MEOHP, MECPP and MEHHP were not observed in MLTC-1 cell medium. The hydrolysis data also implies that diester PAEs-induced steroidogenic effects at high dose (i.e., $100 \mu \mathrm{M})$ are mostly mediated by their parent forms instead of their monoester metabolites. 


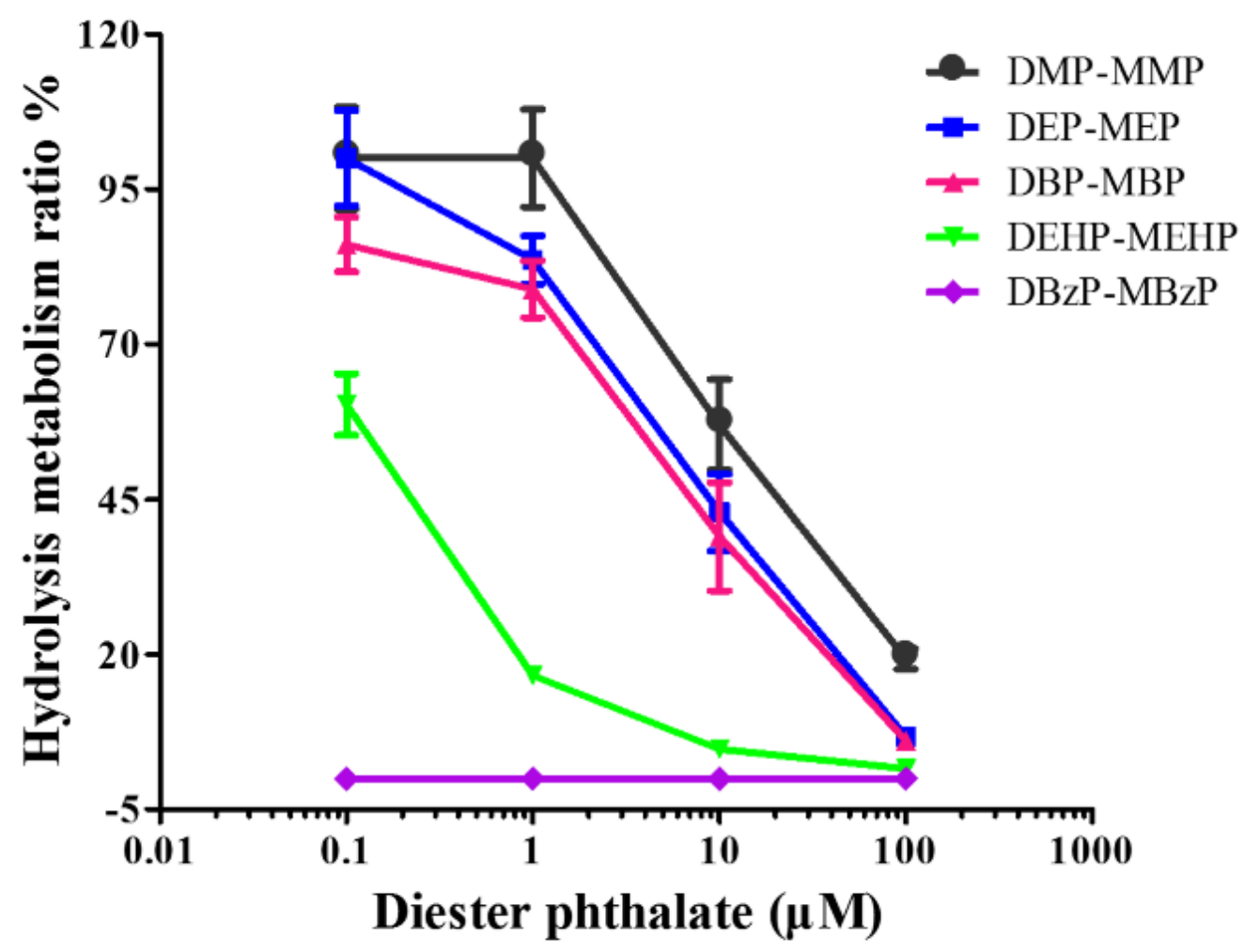

Figure S5. The side chain structure-dependent diester phthalates hydrolyzation.

Five selected equimolar PAEs (DMP, DEP, DBP, DEHP and DBzP) mixed exposure in MLTC-1 cells, the diester phthalates hydrolyzation metabolites found in medium after 48 exposure, as percent of administered concentration. The hydrolyzation rate was calculated by dividing the molar concentration of monoester phthalates by the diester phthalates exposure concentration, respectively. 
Table S7. Different Side Chain Diester Phthalates Have Variant Responses to the Hydrolyzation Enzyme in MLTC-1 Cells with $100 \mu \mathrm{M}$ Mixture Exposure

\begin{tabular}{lcc}
\hline \multirow{2}{*}{ Parent-Metabolite } & \multicolumn{2}{c}{ Percent of metabolite $(\% \pm \mathrm{SD})$} \\
\cline { 2 - 3 } & Negative esterase & Positive esterase \\
\hline DMP-MMP & $19.30 \pm 1.73$ & $59.20 \pm 7.55^{* *}$ \\
DEP-MEP & $6.71 \pm 0.35$ & $34.95 \pm 4.46^{* *}$ \\
DBP-MBP & $6.14 \pm 0.96$ & $64.75 \pm 9.95^{* *}$ \\
DEHP-MEHP & $1.61 \pm 0.14$ & $1.74 \pm 0.26$ \\
DBzP-MBzP & 0 & 0 \\
\hline
\end{tabular}

Asterisks $(* *)$ indicate the significant differences $(p<0.001)$ between the present (positive) and absent (negative) of esterase in the culture medium.

\section{Biphasic steroidogenic effect profiles of PAEs in Leydig cells}

Because DBP may have more toxic characteristics than other $\mathrm{PAEs}^{4}$, its steroidogenic effects on ASD and testosterone generation were initially profiled in detail from 5 to $100 \mu \mathrm{M}$ (Figure S6 A, D). Low-dose DBP exhibited a stimulatory effect on ASD [fold-change comparison to the control $(\mathrm{FC})=1.15(p=0.04)$ for $5 \mu \mathrm{M}$ and FC $=1.41(p<0.001)$ for $10 \mu \mathrm{M}]$, and then a transition point occurred. DBP then generated a significant inhibition on $\mathrm{ASD}$ at least by $50 \mu \mathrm{M}(\mathrm{FC}=0.80, p=0.001)$ and at $100 \mu \mathrm{M}$ DBP, significantly enforced its inhibition on ASD generation $(\mathrm{FC}=0.68, p<0.001)$. DBP inhibited testosterone generation from 25 to $100 \mu \mathrm{M}[\mathrm{FC}=0.82(p=0.03), \mathrm{FC}=$ $0.42(p<0.001)$ and $\mathrm{FC}=0.09(p<0.001)$ for 25,50 and $100 \mu \mathrm{M}$, respectively $)]$, but low-dose stimulation was not observed.

Towards the characterisation of the steroidogenic effects of other PAEs, two key 
doses were set based on the outcomes of DBP experiments, i.e., 10 and $100 \mu \mathrm{M}$. When comparing the capacity of individual PAEs to variously stimulate ASD at $10 \mu \mathrm{M}$ treatments, an increased order of $\mathrm{DMP}(\mathrm{FC}=1.00, p>0.05), \mathrm{DEP}(\mathrm{FC}=1.23, p<0.001)$, $\mathrm{DBP}(\mathrm{FC}=1.41, p<0.001), \mathrm{DBzP}(\mathrm{FC}=1.46, p<0.001)$ and $\mathrm{DEHP}(\mathrm{FC}=1.54, p$ $<0.001$ ) was observed (Figure S6 B, E). For ASD generation at $100-\mu \mathrm{M}$ treatments, DMP or DEP retain their significant ability to stimulate ASD generation. Although DBzP and DEHP are dominated by their diester forms (Figure S5), their stimulatory effects at $100 \mu \mathrm{M}$ were surprisingly retained despite being in their attenuation phases (Figure S6 B). In the 10- $\mu \mathrm{M}$ treatment groups, no effect on testosterone production is observed for all the investigated chemicals. This suggests that ASD regulation may be a more sensitive biomarker than testosterone in response to low-level treatment of PAEs. In the $100-\mu \mathrm{M}$ groups, $\mathrm{DEP}$ acts as a stimulator of testosterone generation $(\mathrm{FC}=1.19$; $p=0.001)$, while $\mathrm{DBzP}$ significantly inhibits production of testosterone $(\mathrm{FC}=0.64 ; p$ $<0.001)$ but to a lesser extent than DBP $(\mathrm{FC}=0.09, p<0.001)$; DEHP also markedly (not significant) inhibits testosterone production $(\mathrm{FC}=0.90 ; p=0.12)$ (Figure $\mathrm{S} 6 \mathrm{~B}, \mathrm{E})$. 

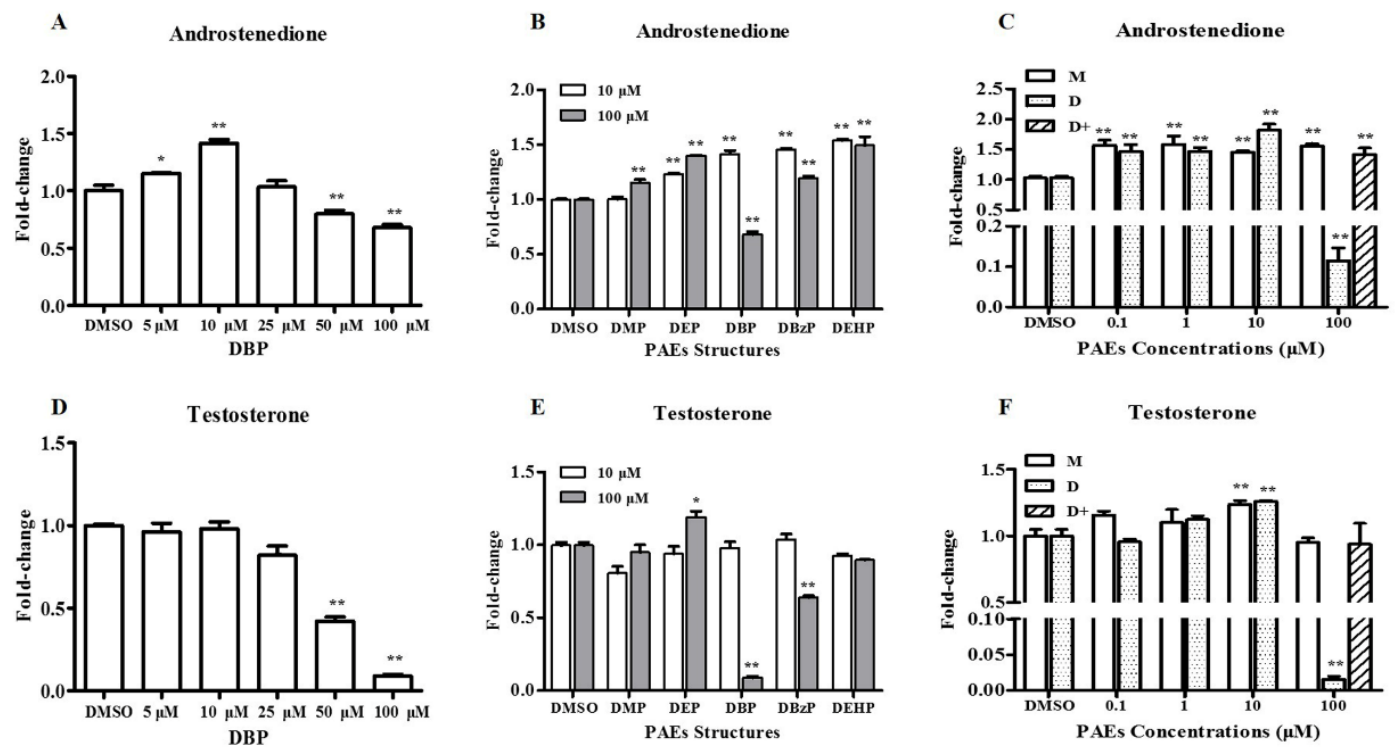

Figure S6. Effects of phthalate exposure for $48 \mathrm{~h}$ on hCG-activated steroid hormone secretion in MLTC-1 cells. (A) DBP vs androstenedione; (B) individual PAEs vs androstenedione; (C) mixed PAEs vs androstenedione; (D) DBP vs testosterone; (E) individual PAEs $v s$ testosterone; and, (F) mixed phthalate $v s$ testosterone.

$\mathrm{M}$ indicates the treatment with mixture of MMP, MEP, MBP MBZP and MEHP; D indicates the mixture treatment of DMP, DEP, DBP DBZP and DEHP; and, D+ indicates exogenous esterase co-treated with diester phthalate mixture at $100 \mu \mathrm{M}$.

The values are expressed as mean $( \pm$ SEM $)$ fold-changes of controls. Data were analysed by one-way ANOVA with Tukey's multiple comparisons test. Asterisks above columns indicate a significant difference in comparison to the control, $* p \leq 0.05, * * p \leq 0.01$.

\section{Steroidogenic responses to PAE mixture exposures in Leydig cells}

Both monoester and diester PAEs mixture tests may simulate some scenarios of real-world human exposure. Firstly, exposed PAEs can rapidly undergo liver metabolism in humans and monoesters are the major functional forms. In relation to 
ASD secretion, when compared with the control group, the entire monoester dose range (from 0.1 to $100 \mu \mathrm{M}$ mixture) significantly stimulated ASD production, while testosterone stimulation only begins to be significant from the $\geq 10-\mu \mathrm{M}$ mixture but begins to be attenuated at the $100-\mu \mathrm{M}$ mixture (Figure S6 $\mathrm{C}$ and $\mathrm{F}$ ). When the exogenous esterase was added in the diester mixture, the diester inhibitory effect is totally reversed for ASD and testosterone production (Figure S6 C and F). This further supports the notion that monoester residuals may have a very limited possibility to act as anti-androgens in real-world human exposure scenarios.

Although each individual diester (except DBP) at $100 \mu \mathrm{M}$ only exhibits androgenic effects, their mixtures may replicate an extremely high human exposure scenario, wherein both ASD and/or testosterone secretion are inhibited in MLTC-1 model. However, the data from exogenous esterase addition may support the notion that human metabolism can mitigate or reverse anti-androgenic effects to androgenic. The low-dose (at least $\leq 10 \mu \mathrm{M}$ ) diester exposure suggests that some minimal PAEs exposure scenarios still generate androgenic effects. The data also suggests that generally, diester forms more readily act as anti-androgens than monoesters. Because of the much lower detection frequency of $\mathrm{MBzP}$ (BBzP can also be the hydrolysed to $\mathrm{MBzP}$ ) in our participant urine implied a much lower-level DBzP exposure, the MLTC-1 cells results factually support the present observations that PAEs exposure can readily transition from androgenic to anti-androgenic mechanism of action. 


\section{References}

1. Liu, L.; Bao, H.; Liu, F.; Zhang, J.; Shen, H., Phthalates exposure of Chinese reproductive age couples and its effect on male semen quality, a primary study. Environ Int. 2012, 42, 78-83.

2. Barr, D. B.; Silva, M. J.; Kato, K.; Reidy, J. A.; Malek, N. A.; Hurtz, D.; Sadowski, M.; Needham, L. L.; Calafat, A. M., Assessing human exposure to phthalates using monoesters and their oxidized metabolites as biomarkers. Environ Health Perspect 2003, 111, 1148-1151.

3. Liu, L.; Wang, H.; Tian, M.; Zhang, J.; Panuwet, P.; D'Souza, P. E.; Barr, D. B.; Huang, Q.; Xia, Y.; Shena, H., Phthalate metabolites related to infertile biomarkers and infertility in Chinese men. Environ Pollut. 2017, 231:291-300.

4. Howdeshell, K. L.; Wilson, V. S.; Furr, J.; Lambright, C. R.; Rider, C. V.; Blystone, C. R.; Hotchkiss, A. K.; Gray, L. E., Jr., A mixture of five phthalate esters inhibits fetal testicular testosterone production in the sprague-dawley rat in a cumulative, dose-additive manner. Toxicol Sci. 2008, 105, 153-65. 